\title{
Application of Surface Roughness Data for the Evaluation of Depth Profile Measurements of Nanoscale Multilayers
}

\author{
András Bartók, ${ }^{a}$ Attila Csik, ${ }^{\mathrm{c}}$ Kálmán Vad, ${ }^{\mathrm{c}}$ György Molnár, ${ }^{\mathrm{b}}$ Enikő Tóth-Kádár, \\ and László Péter ${ }^{\mathrm{a}, *, \mathrm{z}}$
}

${ }^{a}$ Research Institute for Solid State Physics and Optics and ${ }^{b}$ Research Institute for Technical Physics and

Materials Science, Hungarian Academy of Sciences, 1525 Budapest, Hungary

${ }^{c}$ Nuclear Research Institute, Hungarian Academy of Sciences, 4031 Debrecen, Hungary

\begin{abstract}
A secondary neutral mass spectrometric (SNMS) depth profile study of electrodeposited Co/Cu multilayers was performed. Depth profile measurements were performed both in the conventional way (i.e., starting the sputtering from the final deposit surface) and in the reverse manner (i.e., detaching the multilayers from the substrate and starting the analysis from the substrate side, which was very smooth as compared to the final deposit surface). The latter method could yield significantly larger intensity fluctuations in the SNMS spectra. Surface roughness data were measured with atomic force microscopy (AFM) for multilayers with different bilayer numbers but otherwise exhibiting the same layer structure as those used for the depth profiling. The experimental AFM surface roughness evolution was used to calculate the result of the depth profile measurements quantitatively. An excellent agreement was obtained between this calculation and the SNMS measurements. It was shown that the decrease in the intensity fluctuations during the depth profile analysis stems mainly from the increase in surface roughness of the samples studied, especially in the conventional sputtering mode. It was also concluded that the thickness fluctuation of the entire multilayer deposit and that of each layer are strongly correlated.

DOI: $10.1149 / 1.3133182$
\end{abstract}

Nanoscale magnetic/nonmagnetic multilayers are in the forefront of materials research since the discovery of giant magnetoresistance (GMR) in these nanostructures. ${ }^{1,2}$ Multilayers are mostly produced by physical methods (evaporation, sputtering, and molecular beam epitaxy), some of them applying a fairly expensive high vacuum system. The feasibility of the electrodeposition of metallic magnetic/ nonmagnetic multilayers with GMR was demonstrated ${ }^{3}$ a few years after the discovery of the phenomenon. Although electrodeposition has long been considered as a possible low cost alternative to the physical sample preparation techniques, the quality of the electrodeposited (ED) multilayers is still inferior to their physically produced analogs. The literature of the ED multilayer films with GMR amounts to some 140 papers, ${ }^{4-6}$ but very little is known about why the sample quality, especially GMR, cannot achieve the properties of the samples prepared by physical methods.

Although depth profile analysis is a very efficient tool for the characterization of element distribution and the interface quality of ED multilayer samples, only a few studies were published hitherto. Basile et al. ${ }^{7}$ studied the depth profile of $\mathrm{ED} \mathrm{Co} / \mathrm{Cu}$ sandwiches by Auger electron spectroscopy. They found that the observed interface width of approximately $20 \mathrm{~nm}$ is an inherent feature of the sample itself rather than the artifact of the sputtering method used for the depth profiling (although the sputtering also leads to some intermixing at a smaller depth scale $\left.{ }^{8,9}\right)$. According to Tokarz et al., ${ }^{10}$ the interface width of $\mathrm{ED} \mathrm{Cu}(200 \mathrm{~nm}) / \mathrm{Ni}(200 \mathrm{~nm})$ bilayers can also be estimated as being $20-30 \mathrm{~nm}$, as shown by their secondary-ion mass spectrometric depth profile data. Later, Péter et al. ${ }^{11}$ and Katona et al. ${ }^{12}$ published the depth profile analysis of $\mathrm{ED} \mathrm{Co} / \mathrm{Cu}$ and $\mathrm{Co}-\mathrm{Ni} / \mathrm{Cu}$ multilayers. The interface width was estimated to be in the same range as in the study of Basile et al. In the papers listed above, the thickness of a single layer was within the $20-100 \mathrm{~nm}$ range. Gupta et al. studied $\mathrm{Co} / \mathrm{Cu}$ layers with a $6.8 \mathrm{~nm}$ bilayer period with X-ray photoelectron spectroscopy (XPS) ${ }^{13}$ and found an antiphase composition oscillation of $\mathrm{Co}$ and $\mathrm{Cu}$; nevertheless, the resolution was limited by the large probe depth of the XPS method. The depth profile analysis of thinner ED layers still remained a challenge with any technique.

The interface width on the order of $20 \mathrm{~nm}$, as obtained in earlier depth profile studies of ED multilayers, must surely be an apparent value. All major reviews on ED multilayers ${ }^{4-6}$ agree that the elec-

* Electrochemical Society Active Member.

z E-mail: lpeter@szfki.hu trodeposition of magnetic/nonmagnetic multilayers with $2-5 \mathrm{~nm}$ layer thicknesses is fairly easy. Electrical and magnetotransport properties clearly indicate both the presence of a layer structure with a much lower interface width as that shown by the depth profiles and the dominance of regular parts over less ordered regions within the samples. From a structural viewpoint, the occurrence of satellite reflections in the large-angle X-ray diffractograms ${ }^{10,13-22}$ and peaks in the small-angle X-ray diffractograms ${ }^{23-26}$ also underpins the assumption that the real interface width in ED multilayers must be small compared to the layer thickness itself, and the apparent $20 \mathrm{~nm}$ interface width should be explained with something else. Nevertheless, the fairly weak control of the interface quality and the layer thickness fluctuations on the large scale are clearly shown in relevant literature by the large variation in the satellite reflection intensity, the critical layer thickness above which satellites are observed at all, and the number of satellite reflections observed. Transmission electron microscopy (TEM) also revealed a clear layer structure for ED multilayers ${ }^{16,19,22,26-32}$ with repeat lengths well below $10 \mathrm{~nm}$.

In some cases, the observations were compared to model calculations concerning interdiffusion and interface roughness, too. It was revealed by the comparison of low angle $\mathrm{X}$-ray diffraction measurements and calculated scattering intensities ${ }^{26}$ that the interface roughness alone cannot be responsible for the features observed in the diffractograms. Nallet et al. concluded for $\mathrm{ED} \mathrm{Co}-\mathrm{Cu} / \mathrm{Cu}$ multilayers ${ }^{29}$ that neither interdiffusion nor roughness should be responsible for an uncertainty larger than two atomic layers. Therefore, there must be a chance to observe a sharp layer structure with direct depth profiling, too.

Secondary neutral mass spectrometry yields the composition of the surface layer removed, as opposed to several other methods frequently used for the depth profile analysis. The advantages of SNMS, ${ }^{33,34}$ the comparison with other depth profile analysis methods, ${ }^{35}$ and future perspectives ${ }^{36}$ can be found in various reviews. The high depth resolution capability of SNMS gives a chance for detecting subtle features of ED multilayers, too.

In this work, we made an attempt to introduce a sample preparation technique for the depth profile analysis that makes it possible to start depth profiling at a surface with a mean roughness below 1 $\mathrm{nm}$. The results were correlated with a direct surface roughness analysis by using the atomic force microscopy (AFM) technique at different stages of multilayer electrodeposition. 


\begin{tabular}{|c|c|}
\hline $\begin{array}{l}\text { Sample } \\
\text { type }\end{array}$ & $\begin{array}{l}\text { Layer } \\
\text { structure }\end{array}$ \\
\hline A & $\mathrm{Si} / \mathrm{Cr}(20 \mathrm{~nm}) / \mathrm{Cu}(20 \mathrm{~nm}) / /[\mathrm{Co}(5.4 \mathrm{~nm}) / \mathrm{Cu}(4.4 \mathrm{~nm})] \times 7$ \\
\hline B & $\mathrm{Si} / \mathrm{Cr}(5 \mathrm{~nm}) / \mathrm{Cu}(20 \mathrm{~nm}) / /[\mathrm{Co}(7.0 \mathrm{~nm}) / \mathrm{Cu}(5.5 \mathrm{~nm})] \times 7$ \\
\hline $\mathrm{C}$ & $\mathrm{Si} / \mathrm{Cr}(5 \mathrm{~nm}) / \mathrm{Cu}(20 \mathrm{~nm}) / /[\mathrm{Co}(8.7 \mathrm{~nm}) / \mathrm{Cu}(7.8 \mathrm{~nm})] \times 6$ \\
\hline $\mathrm{D}$ & $\mathrm{Si} / \mathrm{Cr}(5 \mathrm{~nm}) / \mathrm{Cu}(20 \mathrm{~nm}) / /[\mathrm{Co}(11.0 \mathrm{~nm}) / \mathrm{Cu}(9.0 \mathrm{~nm})] \times 5$ \\
\hline
\end{tabular}

\section{Experimental}

Materials. - Substrates for all experiments were fabricated from (100)-oriented n-type Si wafers. A 5-20 nm thick $\mathrm{Cr}$ adhesive layer and then a $20 \mathrm{~nm}$ thick $\mathrm{Cu}$ conductive seed layer were evaporated onto the Si wafer. These layers provided a good adhesion for the duration of the electrodeposition procedures, but the detachment of thick deposits from the substrate by a peeling-off process was also possible.

All chemicals used were of analytical grade and purchased from Reanal (Hungary). Electrolyte solutions were prepared with double distilled water. The solution for the deposition of $\mathrm{Co}-\mathrm{Cu} / \mathrm{Cu}$ multilayers contained the following components: $\mathrm{CoSO}_{4}(0.80 \mathrm{~mol} / \mathrm{L})$, $\mathrm{CuSO}_{4}(0.015 \mathrm{~mol} / \mathrm{L}), \mathrm{H}_{3} \mathrm{BO}_{3}(0.20 \mathrm{~mol} / \mathrm{L})$, and $\left(\mathrm{NH}_{4}\right)_{2} \mathrm{SO}_{4}(0.20$ $\mathrm{mol} / \mathrm{L})$.

Electrodeposition.- Electrodeposition was carried out by using a tubular cell. ${ }^{21}$ The $\mathrm{Si} / \mathrm{Cr} / \mathrm{Cu}$ substrate was facing upward. The tubular cell geometry provided an even current distribution over the entire cathode surface. The nominal sample surface area was constant $\left(1.5 \mathrm{~cm}^{2}\right)$, but the real surface area showed some $15 \%$ variation from sample to sample due to the difference in the compression of the silicon rubber gasket between the body of the cell and the substrate. The electrodeposition of the multilayer samples was always terminated with the $\mathrm{Cu}$ layer to reduce the corrosion of the sample after finishing the deposition.

$\mathrm{A} \mathrm{Cu}$ counter electrode was immersed into the electrolyte close to the top of the cell. A saturated calomel electrode was connected as a reference electrode to the cell via a liquid junction (Luggin capillary) positioned at a fixed distance from the cathode. Potential values refer to this electrode throughout this work.

A computer-controlled Electroflex 453 potentiostat/galvanostat was used to deposit the multilayers. This instrument enabled us to apply either potential control or current control for the deposition of the alternating layers of the samples. The Co-rich layers were deposited at a $-60 \mathrm{~mA} / \mathrm{cm}^{2}$ constant current density with a predefined pulse duration, and the $\mathrm{Cu}$ layers were produced at a $-0.6 \mathrm{~V}$ constant potential by monitoring the charge passed through the cell. The nominal layer structure of the samples is summarized in Table I. The interface between the substrate (evaporated layers) and the ED samples is marked with a double slash, whereas layers produced with the same method are separated with a single slash only. The current efficiency was $100 \%$ for the deposition of $\mathrm{Cu}$, whereas it was negligibly less than $100 \%$ during the deposition of Co, as shown by the current efficiency calculation for a large set of samples prepared from an electrolyte of identical composition. ${ }^{20}$

When the deposition of the multilayer samples was completed, the bath used was removed from the cell as soon as it was possible (typically within $30 \mathrm{~s}$ ). Then, the cell was repeatedly rinsed with double distilled water, and the sample cleaning was finished by washing it with small portions of ethanol three times. The fast change of solution and the thorough rinse in ethanol prevented the oxidation of the sample surface to a large extent, leading to a mirrorlike surface similar to the $\mathrm{Si} / \mathrm{Cr} / \mathrm{Cu}$ substrate by visual observation. After drying the samples in air, the sample surface was protected from mechanical contaminations to provide clean conditions for either the surface roughness study or the depth profile analysis.
Multilayer samples intended for the reverse depth profile analysis were coated with an ED Ni layer immediately after the deposition of the multilayers. In this procedure, the electrolysis cell was not disassembled; just the electrolyte was changed. The Ni cover layer was produced by using a conventional Watts-type bath operated at a low current density $\left(-6 \mathrm{~mA} / \mathrm{cm}^{2}\right)$, hence obtaining a relatively stressfree, shiny deposit with uniform thickness. This Ni layer was strong enough to prepare self-supporting samples if its thickness exceeded $2 \mu \mathrm{m}$.

Surface roughness analysis. - The surface roughness parameters were calculated from the analysis of AFM images. These measurements were made with a MultiMode AFM from Veeco Metrology in the noncontact mode with a resolution of $256 \times 256$ pixels. In a few cases, the measurement was also carried out in the contact mode, and the results were identical, with a little difference in the noise level. The diameter of the end of the AFM tip was 20-30 nm according to the manufacturer's specification. The symmetry of the height distribution curves was distorted, probably because the diameters of the holes on our sample surface were in some cases less than the radius of the AFM tip used for the measurements. ${ }^{37}$

For a comparison of the roughness of various samples, measurements were taken over $5 \times 5 \mu \mathrm{m}$ rectangular areas of the sample surface. To check the independence of the roughness parameters measured from the dimension of the area scanned, ${ }^{38}$ the edge of the square scanned was varied from 2.5 to $20 \mu \mathrm{m}$. The analysis of the AFM picture was performed with the Gwyddion software. ${ }^{39}$

For the roughness calculation, a low pass two-dimensional Fourier filter was first applied to remove the high frequency noise from the pictures, and then the height distribution functions were calculated. These distributions can be perfectly fitted by a Gaussian function; hence, the full width at half-maximum (fwhm) is a relevant and sufficient parameter to describe the roughness of the fitted curves. Because the direction of the lateral motion of the AFM tip was not necessarily parallel to the mean sample surface, the polynomial background correction procedure of the analysis software was used for this data correction where it was needed.

Depth profile analysis. - SNMS depth profile measurements were carried out by an instrument of the type INA-X (SPECS $\mathrm{GmbH}$, Berlin). Secondary neutral mass spectrometry is a destructive technique because the sample surface is sputtered by an ion beam, which was $\mathrm{Ar}^{+}$in this case. The material abraded by sputtering consisted mostly of neutral atoms, which were detected after postionization using electron cyclotron wave resonance plasma. As opposed to several other surface analysis techniques, in the SNMS measurements practically the total amount of the material removed was analyzed, and neither the ionization probability during the sputtering itself (like in secondary-ion mass spectrometry) nor the difference in the bulk and surface layer compositions (like in XPS) was important. This eliminates the matrix effect and makes it possible to carry out an accurate quantitative elemental analysis.

The intensity vs sputtering time function (i.e., the raw data set obtained in the measurement) was transformed into the molar fraction vs depth curves in two steps. In the first step of the procedure, the concentrations of the elements were calculated from these intensities using the relative sensitivity factors of the elements. In the second step, the time scale was converted into a depth scale by using the sputtering rates of each element that were determined previously by measuring the sputtering time and the resulting crater depth under the same sputtering condition. Hence, a true depth scale can be obtained for all compositions detected. The entire procedure is described in detail in a previous work. ${ }^{11}$

Two opposite sputtering directions were applied in the present depth profile measurements. First, for conventional depth profile analysis (where samples still on their substrate were studied as obtained after the electrodeposition) the sputtering of the sample was started from the final surface; i.e., the last layer deposited was sputtered first ("top-to-bottom" etching). Second, the reverse depth profile analysis was carried out with the following protocol. The 

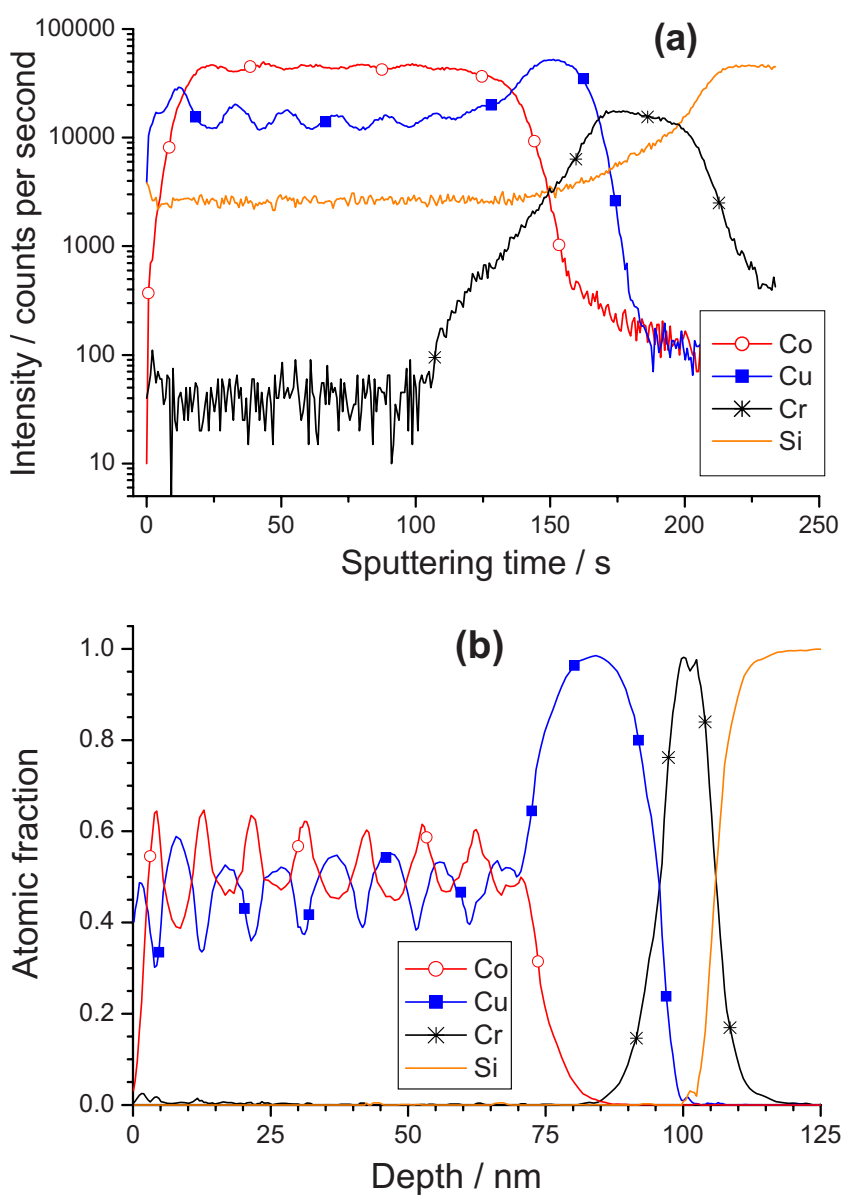

Figure 1. (Color online) Conventional depth profile of a type-A multilayer sample.

multilayer sample coated with the supporting Ni layer was detached from the substrate. The back side of the Si wafer was scratched parallel to the crystallographic axis. The sample was bent by pressing the wafer so that the deposit was at the concave side. Therefore, the Si wafer could be broken, but the Ni support prevented the sample from being damaged. After breaking the Si wafer, the sample could be peeled off from the substrate very easily. The separation took place at the $\mathrm{Si} / \mathrm{Cr}$ interface at fairly large areas. Although the evaporated $\mathrm{Cr} / \mathrm{Cu}$ layer was damaged at some areas, it was always possible to find a large enough spot on the sample where the $\mathrm{Cr}$ layer entirely covered the multilayer deposit. By following this sample preparation procedure, the depth profile analysis could be started from the Cr layer ("bottom-to-top" etching), whose surface roughness was comparable to the polished surface of the Si wafer $(\sim 1 \mathrm{~nm})$.

The sputtering in the SNMS instrument was carried out for a round-shaped area with a diameter of 2-3 $\mathrm{mm}$. The calculation method of the composition vs depth function from the detected intensity vs time function was given elsewhere. ${ }^{11}$ The calculation of the apparent interface width from the SNMS depth profiles was performed in accordance with the method shown in Fig. 3b of Ref. 11. Briefly, the tangent line was drawn to the inflection point of the concentration vs depth function, and the difference in the intercepts with the previous and subsequent baselines was calculated.

\section{Results}

Comparison of conventional and reverse depth profile analyses.-A conventional SNMS depth profile was obtained for a type-A multilayer. The resulting intensity vs time curve is shown in Fig. 1a. At the beginning of the sputtering, the fluctuation of the
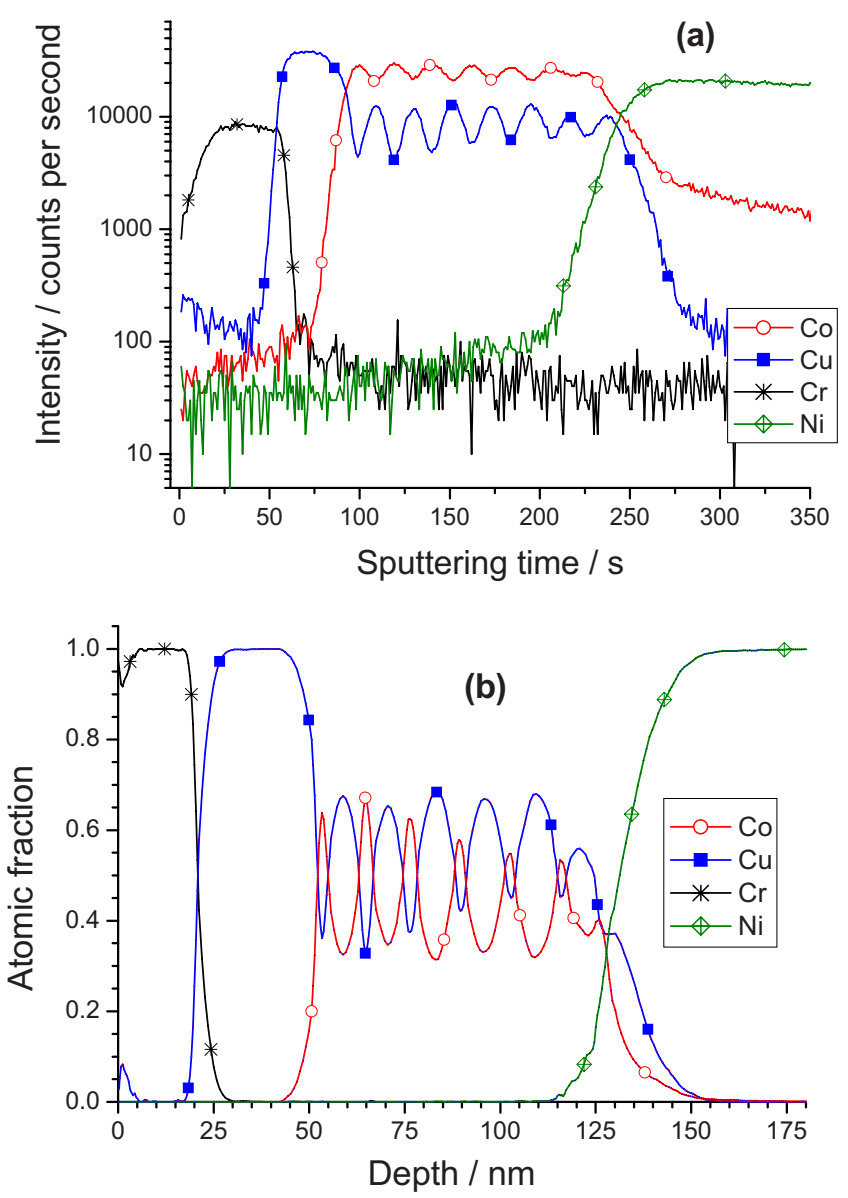

Figure 2. (Color online) Reverse depth profile of a type-A multilayer sample.

intensities related to the main components of the multilayer (i.e., Co and $\mathrm{Cu}$ ) can be seen, but the intensity fluctuation decays so fast that the last bilayer (closest to the substrate) cannot be resolved. During the sputtering of the multilayer structure, the intensity fluctuation of the $\mathrm{Cu}$ signal is $38 \%$, whereas it amounts to $10 \%$ only for Co. The corresponding molar fraction vs depth function is displayed in Fig. 1b. The bilayer repeat distance established from the latter set of curves is $9.9 \mathrm{~nm}$, which is identical to the $9.8 \mathrm{~nm}$ nominal value well within error. The thickness of the $\mathrm{Cr}$ layer as observed in the depth profile also agrees with the nominal substrate layer structure. Below a repeat distance of $10 \mathrm{~nm}$, the conventional depth profile analysis could not reveal the layered structure of the samples.

A multilayer with the same structure was also investigated by the reverse depth profiling method. The result of the SNMS measurement is shown in Fig. 2. The intensity fluctuation in Fig. 2a is much higher than that for the conventional depth profiling method. The intensity fluctuation of the $\mathrm{Cu}$ signal increased to $62 \%$ (from $38 \%$ ), and a similar increase was observed for the Co signal as well $(24 \%$ instead of $10 \%$ ).

It is evident from Fig. $1 b$ that the layer structure is observed in a faded manner because the apparent molar fraction in the middle of the layers is still by far lower than 1 . The apparent interface width as a function of the sputtering depth is shown in Fig. 3. In the case of conventional sputtering, the apparent interface width obtained is comparable to the layer thickness itself; therefore, large SNMS intensity fluctuations cannot be expected. While the apparent interface width steadily increases with sputtering depth in the conventional depth profile analysis, it achieves approximately $3 \mathrm{~nm}$ and remains constant for the entire sputtering process in the reverse sputtering method. This $3 \mathrm{~nm}$ apparent interface width is better than the $5 \mathrm{~nm}$ 


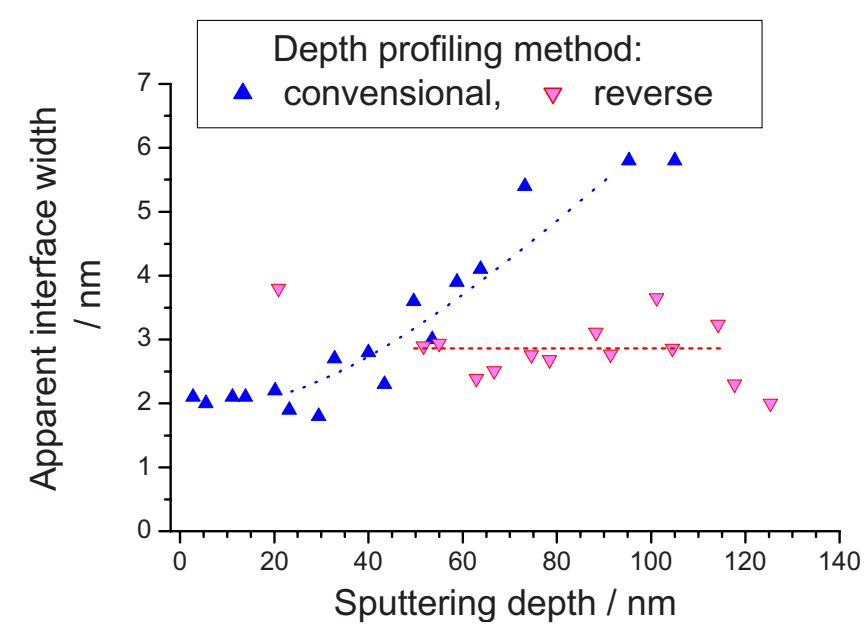

Figure 3. (Color online) Apparent interface width for type-A multilayer samples analyzed in conventional and reverse manner as a function of the sputtering depth.

width found formerly for the conventional sputtering direction. ${ }^{11}$ Because in the reverse depth profiling the starting surface is almost atomically smooth, the $3 \mathrm{~nm}$ apparent interface width can be attributed to the sum of the natural interface width produced by the electrodeposition, the apparent interface width increment due to the layer undulation, and the artifact of the sputtering process itself.

Reverse depth profile analysis of multilayers with various layer thicknesses. - When the bilayer thickness was around $10 \mathrm{~nm}$, the periodicity of the multilayers could be easily elucidated from the depth profile analysis performed by either the conventional or the reverse method. However, the SNMS intensity fluctuation remained well below the value that one can expect from either the electrochemical sample preparation parameters or the direct observation of the layer structure with TEM. This can be caused by at least two phenomena, namely, the loss in the interface sharpness and the roughness of the sample surface (or even both). Figure 4 illustrates how the low interface sharpness and the increase in surface roughness during the deposition may affect the depth profile observed experimentally if one of the effects is predominant.

The scheme in Fig. 4 is valid only if the layer thickness is significantly larger than the widening of the signal caused by the sputtering process itself. Hence, there is a chance that the lower-thanexpected intensity fluctuation can be attributed to one of the reasons taken into account in Fig. 4 if multilayers with larger and larger layer thicknesses are analyzed. For this reason, multilayers of types $\mathrm{B}, \mathrm{C}$, and $\mathrm{D}$ were prepared for the reverse depth profile analysis (see Table I). The results are shown in Fig. 5. All depth profile curves show a very sharp layer interface at the $\mathrm{Cr} / \mathrm{Cu}$ boundary, and the molar fraction of $\mathrm{Cu}$ is very close to or even equal to 1 when the $\mathrm{Cu}$ seed layer of the substrate is being sputtered. However, the intensity of both $\mathrm{Cu}$ and $\mathrm{Co}$ decays fast as the sputtering depth increases. The qualitative evaluation of the depth profiles indicates that the shape of the envelope curve of the depth profile functions agrees with the assumption that the uneven layer thicknesses and the resulting layer corrugation may be responsible for the loss of the SNMS fluctuation intensity with increasing sputtering depth.

Surface roughness measurements. - To estimate the impact of the increase in the sample surface roughness on the experimental depth profile curves, AFM measurements were carried out for the final surface of various ED samples. The saturation surface roughness of both the $\mathrm{Si}$ wafer and the $\mathrm{Si} / \mathrm{Cr} / \mathrm{Cu}$ substrate was between 0.5 and $1.2 \mathrm{~nm}$, which is the limit of the height resolution of the AFM instrument.

The analysis of a series of AFM measurements for a B-type multilayer sample is shown in Fig. 6. It can be seen in the curves of

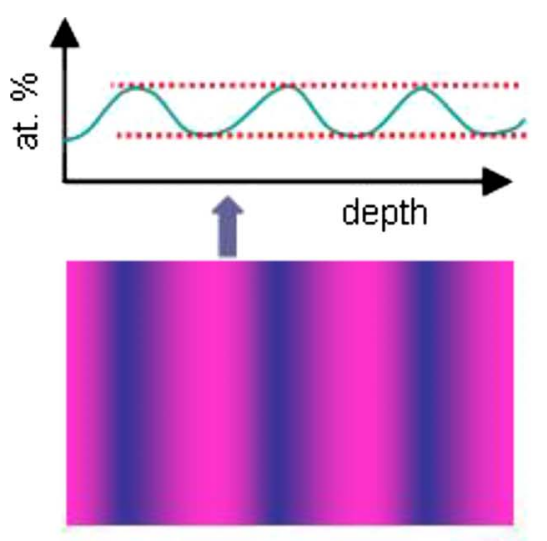

Expected depth profile

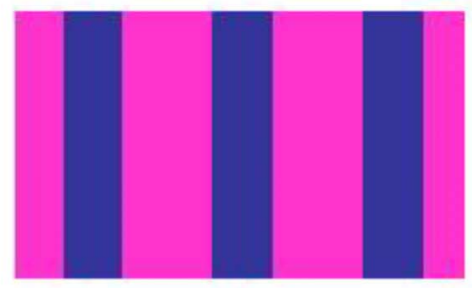

Nominal

layer

structure

(ideal case)

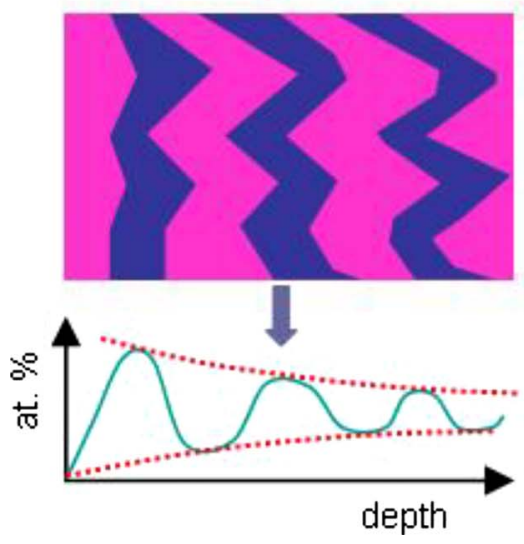

Structure

distortion by

intermixing

Structure

distortion by

roughening

Expected depth

profile

Figure 4. (Color online) Impact of the fading of layer interfaces and that of the surface roughening on the experimental depth profile data.

Fig. 6 that at the lateral length scales chosen for the AFM study, the height distribution curve is independent of the length scale. All curves could be fitted with a Gaussian distribution. The slight asymmetry of the curves (more likely deviation from the average at extremely large heights than in deep cavities) could be detected for practically all curves. However, this asymmetry amounts to about $1 \%$ of all points measured. The shapes of the height distribution functions were all very similar to those published for ED Co and $\mathrm{Cu}$ samples $^{40}$ of similar thicknesses.

To estimate the rate of the surface roughness increment during the electrodeposition, several samples were produced with identical layer thicknesses as samples of the types B, C, and D but with reduced bilayer numbers. Then, the surface roughness of the multilayers was measured as a function of the bilayer number. The surface roughness of the samples increases with the sample thickness at approximately the same rate for all three sample types. In the next section, these roughness vs depth curves are displayed as functions of the total thickness of the metallic coating on the Si wafer (including the sputtered $\mathrm{Cr} / \mathrm{Cu}$ layer) to bring AFM roughnesses and depth profiles to the same depth scale.

AFM was also used to assess the surface roughness of the samples investigated with the reverse depth profiling method. After detaching the metallic coating (seed layer + ED multilayer + ED $\mathrm{Ni}$ layer) from the $\mathrm{Si}$ wafer, the $\mathrm{Cr}$ surface of the detached sample was studied. Figure 7 shows typical linescans obtained for both 

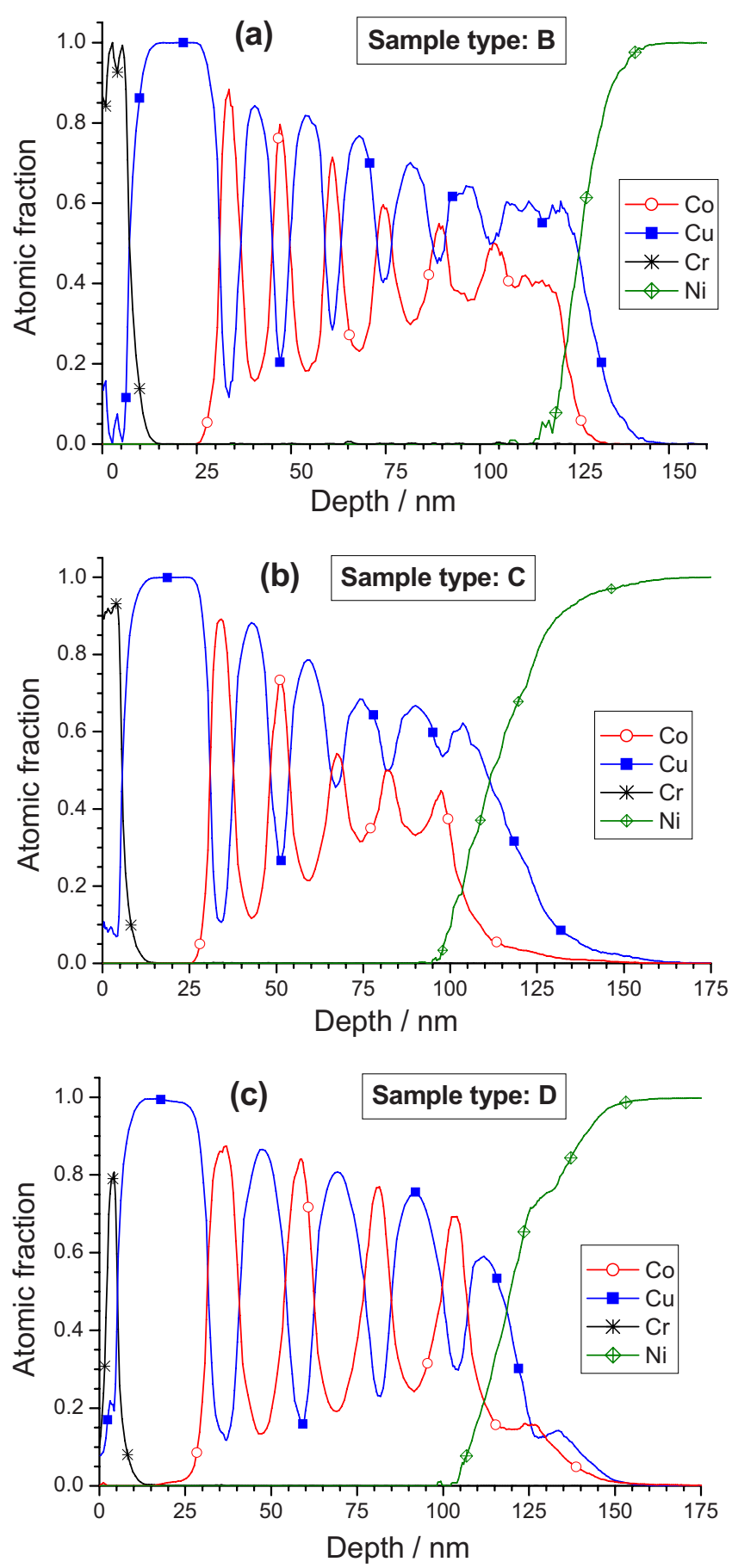

Figure 5. (Color online) Reverse depth profile of multilayer samples of types B, C, and D.

smooth and damaged sample surfaces. When the seed layer on the $\mathrm{Si}$ wafer was defective, the surface of the detached sample became stepwise, and the step height was comparable to the nominal thickness of the $\mathrm{Cr} / \mathrm{Cu}$ seed layer. Only areas of detached samples with a continuous $\mathrm{Cr}$ layer were appropriate for the reverse depth profile analysis where the mean roughness was below $1 \mathrm{~nm}$.

Calculation of the results of reverse depth profile analysis.For the estimation of the results of the depth profile analysis, it was assumed that the decay in the SNMS signal intensity fluctuation was caused solely by the undulation of the layer interfaces, which was the consequence of the uneven layer thicknesses. The probability of the distance of the layer interface from the substrate was described

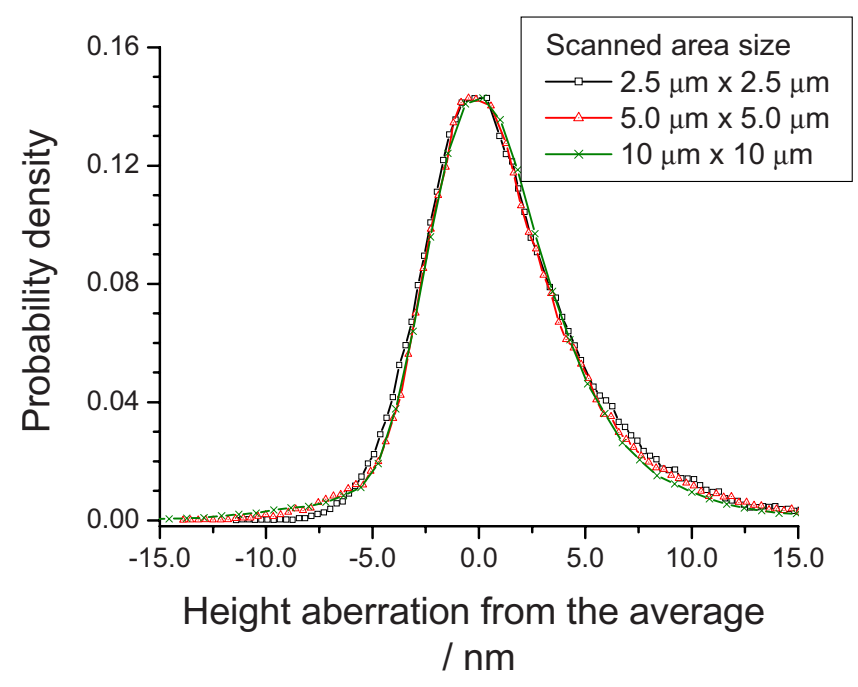

Figure 6. (Color online) Height distribution curves for a B-type multilayer deposit as elucidated from AFM measurements of various length scales.

with a Gaussian function. This approximation was based on the result of the AFM measurement. It was important to choose an analytical function for the calculation, with the help of which the numerical integration could be performed easily. Then, the calculated distribution of component $i\left(y_{\mathrm{calc}, i}\right)$ was obtained as a convolution of the nominal depth profile function of the same component $\left(y_{\mathrm{nom}, i}\right)$ and the probability density function $(G)$

$$
y_{\text {cal }, i}(x)=\int_{-\infty}^{\infty} y_{\text {nom }, i}\left(x^{\prime}\right) G\left(x^{\prime}, x, \sigma(x)\right) d x^{\prime}
$$

The nominal molar fraction $\left(y_{\text {nom }}\right)$ is a square-wave function that describes the depth profile as if all interfaces were planar and parallel to the original substrate surface. For instance, $y_{\text {nom, } \mathrm{Cu}}=1$ when $d_{\mathrm{Cr}+\mathrm{Cu}}+k\left(d_{\mathrm{Cu}}+d_{\mathrm{Co}}\right)<d<d_{\mathrm{Cr}+\mathrm{Cu}}+k\left(d_{\mathrm{Cu}}+d_{\mathrm{Co}}\right)+d_{\mathrm{Cu}}$, where $k$ is an integer that counts the full periods from the substrate. Therefore, all deviation of the shape of layer interfaces from planarity is attributed to the surface roughness and is comprised of the function $G$. By using the height distribution function $G$, the shape of the surface does not need to be taken into account; it is enough to treat its statistical description.

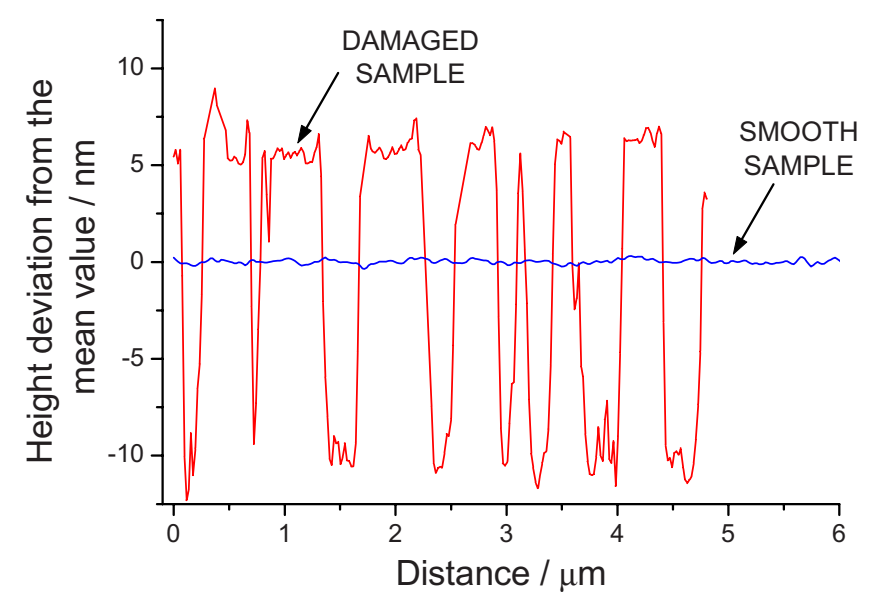

Figure 7. (Color online) Comparison of AFM line scans recorded for the substrate side surface of a good and a damaged sample peeled off from the Si wafer. 
In the argument of the Gaussian function $G, x$ is the mean value of the distribution, and the fwhm of the distribution is also taken as a function of the mean value (the latter is determined by the AFM roughness vs sample thickness measurements). In the samples studied every other layer is $\mathrm{Cu}$ and the neighboring layers are composed of a single element only $(\mathrm{Cr}, \mathrm{Co}$, or $\mathrm{Ni})$, therefore, the $y_{\mathrm{Cu}}+y_{\text {other }}$ $=1$ condition is fulfilled for each transient region. The latter equation and the interchangeability of the addition and integration ensure that $\sum_{i=1}^{N} y_{\mathrm{calc}, i}=1$ for all sputtering depth $x$. Therefore, it is enough to display the depth profile function of $\mathrm{Cu}$ only for the assessment of the feasibility of the calculation. The comparison of the experimental and calculated depth profile functions are shown in the bottom part of Fig. 8a-c, while the corresponding surface roughness vs sample thickness functions are displayed in the top part of the same figures. The best agreement of the calculation and the experimental data is obtained if the actual roughness is assumed to be different by about $0.5-2 \mathrm{~nm}$ from the measured data points throughout the thickness range. It is remarkable that the rate of roughness increment with the sample thickness is identical in the data set measured and that used for the calculation of the best fit.

Figure 8a shows that the surface roughness increment at the rate measured is appropriate for the depth profile calculation, although the initial roughness is somewhat different from that of the Si wafer. However, in Fig. 8b and c the $\mathrm{Cu}$ molar fraction in the last layer deposited seems to be abnormally low, and the loss of $\mathrm{Cu}$ cannot be the consequence of any roughness increment. In these cases, the SNMS intensity for Co also decays earlier than expected. It is believed that the weak agreement of the measurement and the calculation at the $\mathrm{Cu} / \mathrm{Ni}$ interface is caused by the corrosion of the sample when the acidic Ni bath already contacted the multilayer sample but the deposition was not yet started. Although the Ni deposition was started soon after filling up the cell with the electrolyte, the presence of dissolved oxygen and the chloride content of the bath can cause such a problem.

The agreement between the calculated and measured data can be achieved only in the case when the $\mathrm{Cu}$ content of the Co layer is also taken into account $\left(y_{\mathrm{Cu}}^{*}\right)$. This value can be calculated from the electrochemical sample preparation data with the following equation

$$
y_{\mathrm{Cu}}=\frac{Q_{\mathrm{Cu}}+Q_{\mathrm{Co}} \eta y_{\mathrm{Cu}}^{*}}{Q_{\mathrm{Cu}}+Q_{\mathrm{Co}} \eta}
$$

where $y_{\mathrm{Cu}}$ is the average $\mathrm{Cu}$ content of the entire deposit, $y_{\mathrm{Cu}}^{*}$ is the $\mathrm{Cu}$ content of the Co layer, $Q$ is the charge passed during the deposition of the layer shown by the lower index, and $\eta$ is the current efficiency for the Co layer deposition. After rearranging Eq. 2, one obtains

$$
y_{\mathrm{Cu}}^{*}=y_{\mathrm{Cu}}-\left(1-y_{\mathrm{Cu}}\right) \frac{Q_{\mathrm{Cu}}}{Q_{\mathrm{Co}} \eta}
$$

Since $y_{\mathrm{Cu}}^{*}$ is obtained as a difference and its value is close to zero, the uncertainty of the determination of $y_{\mathrm{Cu}}^{*}$ is very large. The current efficiency can also be a function of the layer thickness. This is why $y_{\mathrm{Cu}}^{*}$ was also a free parameter in finding the best approximation of the trial function to the experimental depth profile data. If $y_{\mathrm{Cu}}^{*}$ was neglected, the ratio of the $\mathrm{Cu}$ and $\mathrm{Co}$ layer thicknesses obtained from the depth profile approximation was much larger than that expected from the deposition parameters. The best fit was obtained when $y_{\mathrm{Cu}}^{*}$ was $0.020 \pm 0.012$, which agrees with both the SNMS determination of the $\mathrm{Cu}$ content of thick $\mathrm{Ni}-\mathrm{Co}$ layers prepared under similar conditions ${ }^{11}$ and the determination of the $\mathrm{Cu}$ content of the Co layer in a multilayer series prepared from the same bath. ${ }^{20}$

Despite the fact that the bilayer thickness $\left(\Lambda=d_{\mathrm{Co}}+d_{\mathrm{Cu}}\right)$ used for the simulation of the depth profile measurement agreed with the nominal repeat length, the $\mathrm{Cu}$ layer thickness always needed to be overestimated to approach the experimental data with the simulation. This discrepancy could not be resolved. Some possible reasons are listed in the Discussion.
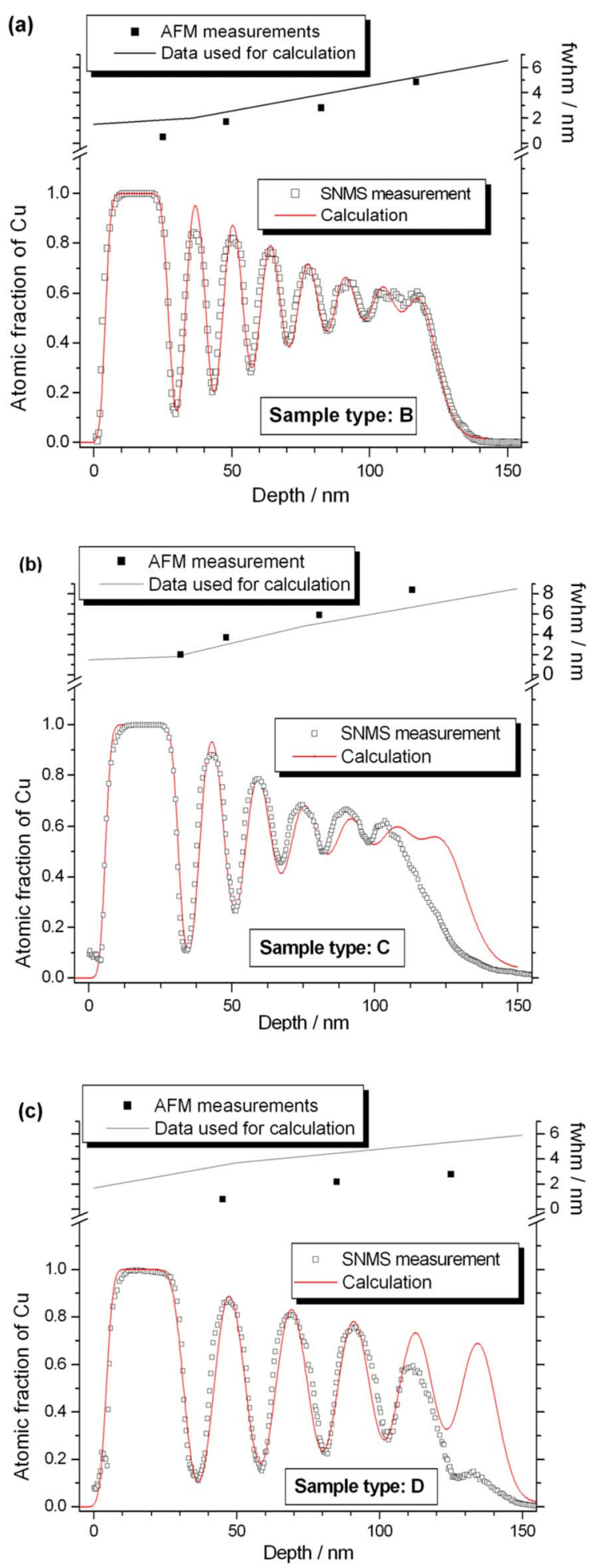

Figure 8. (Color online) Comparison of the measured and calculated depth profile data for various multilayers (bottom parts). The top part of the figures shows the mean AFM roughness measured at the same total sample thickness with individual symbols, and the continuous line is the roughness vs depth function used to achieve the best fit of the calculation to the experimental "reverse" depth profile data.

\section{Discussion}

The length scales of the samples and of the measurements range more than about 6 orders of magnitude. These characteristic distances are summarized in Table II. 


\section{Table II. Summary of the characteristic thicknesses and distances} related to the samples and the experimental methods.

Thickness of a single layer

Total multilayer thickness,

$(5-11) \times 10^{-9} \mathrm{~m}$

including the $\mathrm{Cr} / \mathrm{Cu}$

seed layer

Length scale of the AFM study

Diameter of the area used for depth

profile measurement
$(1.1-1.5) \times 10^{-7} \mathrm{~m}$

$(2.5-10) \times 10^{-6} \mathrm{~m}$

$2 \times 10^{-3} \mathrm{~m}$

The periodicity (i.e., bilayer thickness) of the ED multilayers studied in this work is around $20 \mathrm{~nm}$, while the surface roughness measurements were performed over areas equal to or larger than $2.5 \times 2.5 \mu \mathrm{m}$. Because the characteristic spot diameter for the surface roughness assessment is much larger than either the multilayer periodicity or the total deposit thickness, one can assume that the surface roughness estimation at the length scale applied provides the saturation value of the surface roughness. This is evidenced also by the fact that very similar height distribution functions are obtained for various surface areas scanned. It is of no importance for this study whether the layer growth obeys the normal or the anomalous scaling because for both cases the saturation roughness no longer increases with the enlargement of the area under investigation. ${ }^{38}$ It was also found for both $\mathrm{ED}^{41}$ and chemically deposited ${ }^{42}$ homogeneous metal films and multilayers ${ }^{43}$ that the saturation roughness can be measured at a length scale of $1 \mu \mathrm{m}$ when the total deposit thickness is smaller than about $300 \mathrm{~nm}$. The total thickness of the multilayer deposits in this study is only about one-third of the above value.

The grain size of the ED nanoscale multilayers may vary, depending on all deposition conditions (temperature, electrolyte composition, deposition parameters, and total sample thickness) and on the substrate surface. The grain size estimation from large-angle $\mathrm{X}$-ray diffractograms yields the grain size in the growth direction; hence, it cannot be compared to the correlation length of the surface pattern. Direct TEM observations ${ }^{30,31,44-46}$ can often yield, however, lateral granule diameters of a few hundred nanometers. The AFM surface scan with the characteristic length scale of $2.5 \mu \mathrm{m}$ is surely sufficient to yield the mean roughness of the surface of a $100 \mathrm{~nm}$ thick film, ranging to a statistically large enough number of granules.

The surface area used for the depth profile analysis was about $10^{5}$ times larger than the area used for the surface roughness estimation. However, the roughness observed at the micrometer length scale was appropriate for the estimation of the depth profile observed at the millimeter length scale. This fact indirectly supports the assumption that the AFM measurements gave information about the saturation roughness; otherwise, the calculation could not yield a good estimate of the experimental depth profile data.

The accuracy of the experimental depth profile data strongly depends on whether the peel-off procedure can provide an ideally smooth and defect-free $\mathrm{Cr}$-capped sample. The $\mathrm{Ni}$ adlayer with a thickness larger than $2 \mu \mathrm{m}$ has a large enough mechanical strength to detach the metallic structure from the Si wafer entirely, i.e., without fractures. In some cases the detachment of the $\mathrm{Cr}$ layer was not complete, which is indicated by the presence of $\mathrm{Cu}$ and $\mathrm{Si}$ atoms at the surface already at the beginning of the sputtering; nevertheless, the AFM scan at a micrometer scale indicated a sufficiently smooth surface. This mechanical damage is also a source of the surface roughness that leads to an apparent widening of the interfaces, and it can also be an explanation of why the probability distribution used for the best fit has larger fwhm in some cases than the experimental data. Nevertheless, in most of the cases it was easy to find a spot on the detached sample surface where the depth profile analysis could yield reliable results without the influence of the imperfection caused by the peel-off procedure.

The height distribution functions obtained from the AFM mea-

surements exhibit a slight asymmetry. This is partly because of the finite (nonzero) lateral tip size because it can properly image the elevated areas of the surface (peaks), but cannot follow the shape of the cavities with the same accuracy. It is predicted by theoretical calculations ${ }^{37}$ that as the ratio of the tip radius to the correlation length of the surface features decreases, the AFM roughness tends to underestimate the real value. This can be valid in the case of ED multilayer samples too.

The opportunity for the calculation of the experimental depth profile data is also evidence for one more fact. Namely, the fluctuation of the total sample thickness is strongly correlated to the thickness fluctuation of each layer deposited. If this were not true, then the calculation of the experimental depth profile data could only be performed with the sum of the surface roughness and the thickness fluctuation of an individual layer. In this respect, the multilayer growth pattern suggested in several previous works can also be applied to the multilayer samples of this study (bottom of Fig. 4; see also Fig. 11b of Ref. 32, Fig. 13a of Ref. 44, Fig. 2 of Ref. 45, Fig. 5 of Ref. 47, and Fig. 10.10b of Ref. 48). According to the latter works, the columnar structure formed exhibits elevated areas close to the middle of the crystals, while the shallow areas can be found around the grain boundaries. Similar conclusions were drawn by Shima et al., 22 and the presence of the grooved grain boundary was also one of the explanations for the absence of the coupling between the magnetizations of the neighboring magnetic layers. The direct TEM observations on the columnar growth of the ED multilayers ${ }^{31,45,46}$ agree with such assumptions, although the TEM observations were usually obtained for deposits with much larger total thicknesses than those reported in the present work.

The analysis performed in this study clearly showed that the interface width of about $20 \mathrm{~nm}$ as observed in earlier studies for the conventional depth profiles ${ }^{7,10-12}$ is an artifact that was caused, besides the possible drawbacks of the depth profiling methods themselves, by the fairly large surface roughness developed during the electrodeposition of the samples.

It is worthwhile to discuss the possible reasons why the layer thicknesses in the simulated curves differ significantly from the nominal ones, although the bilayer thickness agrees with the nominal value. When the sputtering front penetrates into the sample, it will cross the layer boundaries because of the corrugation of the layers; in the absence of corrugation, the layer boundaries and the sputtering front would be parallel planes. This must lead to the situation that the surface being sputtered is laterally inhomogeneous, and hence the sputtering rate also varies from spot to spot. However, the calculation of the atomic fractions is based on the assumption that the material studied is laterally homogeneous. Hence, this source of error is the inherent feature of the calculation method that could not be overcome so far. Therefore, one has to accept that the simulation of the depth profile curves can yield a fairly exact value of the bilayer thickness, but the individual layer thicknesses are somewhat misestimated.

In spite of the success of the depth profile data calculation for the reverse sputtering, the fast decay of the fluctuation corresponding to the layered structure in the conventional depth profiling cannot be the result of the initially higher surface roughness alone. It is easy to show that if the fluctuation of the total sample thickness and that of the layer thicknesses are correlated, the fluctuation should show the same decay rate for conventional and reverse sputtering, provided that the original surface profile is conserved during the sputtering process. Instead, the inferior result for the conventional sputtering should be a consequence of the higher starting roughness and the sputtering process together. Hence, this is related to the processes taking place during the sputtering rather than to the thickness fluctuation alone.

Although the simulation of the reverse depth profile curve with the help of the surface roughness evolution function was successful, it could not account for the contribution of the interface roughness to the resolution decay in any sense. We can only say that it is not necessary to include a distortion factor due to the interface rough- 
ness, but there is no definite evidence to completely exclude its impact on the measured data. It is expected that the occurrence of both effects (i.e., increase in the surface roughness accompanied with the loss of interface sharpness) would lead to a faster decay of resolution with sputtering depth.

\section{Conclusions}

A depth profile method was elaborated by detaching the deposits from their substrates. In this reverse depth profile analysis, the sputtering of the sample can be started from the layer that was deposited first, hence eliminating the impact of the roughness of the final surface on the depth profile data measured.

It was shown that the apparent interface width is lower for the reverse depth profiling method than for the conventional one.

A calculation method was developed for taking into account the increase in the surface roughness in the elucidation of the reverse depth profile analysis. It was shown that the experimental depth profile curves can be obtained with the simulation, too, if the rate of the surface roughness increase with sputtering depth in the calculation is the same as that measured experimentally with AFM.

The complementary analysis of the experimental depth profile data and the surface roughness evolution showed that the thickness fluctuation of the entire multilayer sample has to be strongly correlated to that of each layer within the multilayer. If these thickness fluctuations were uncorrelated, the calculation of the depth profile based on the surface roughness could not reproduce the experimental depth profiles.

\section{Acknowledgment}

The authors gratefully acknowledge Áron Pekker for his help in the manipulation of the AFM instrument. Financial support was provided by the Hungarian Scientific Research Fund (OTKA) through grant no. K-60821 and no. K-67866. L.P. thanks Imre Bakonyi for his persistent support and for discussion of the manuscript.

\section{References}

1. M. N. Baibich, J. M. Broto, A. Fert, F. Nguyen Van Dau, F. Petroff, P. Etienne, G. Creuzet, A. Friederich, and J. Chazelas, Phys. Rev. Lett., 61, 2472 (1988).

2. G. Binasch, P. Grünberg, F. Saurenbach, and W. Zinn, Phys. Rev. B, 39, 4828 (1989).

3. M. Alper, K. Attenborough, R. Hart, S. J. Lane, D. S. Lashmore, C. Younes, and W. Schwarzacher, Appl. Phys. Lett., 63, 2144 (1993).

4. W. Schwarzacher and D. S. Lashmore, IEEE Trans. Magn., 32, 3133 (1996).

5. I. Bakonyi and L. Péter, in Magnetic Materials, Processes and Devices VIII, S. Krongelb, Editor, PV 2004-23, p. 227, The Electrochemical Society Proceedings Series, Pennington, NJ (2004).

6. L. Péter and I. Bakonyi, in Electrocrystallization in Nanotechnology, G. Staikov, Editor, p. 242, Wiley-VCH, Weinheim (2006).

7. F. Basile, J. Bergner, C. Bombart, P. Nallet, E. Chassaign, and G. Lorang, Microsc. Microanal. Microstruct., 8, 301 (1997).

8. M. Menyhard, G. Zsolt, P. J. Chen, C. J. Powell, R. D. McMichael, and W. F. Egelhoff, Appl. Surf. Sci., 180, 315 (2001).

9. A. Barna, M. Menyhard, A. Zalar, and P. Panjan, Appl. Surf. Sci., 242, 375 (2005).

10. A. Tokarz, A. Wolkenberg, and T. Przeslawski, J. Electrochem. Soc., 149, C607 (2002).

11. L. Péter, G. L. Katona, Z. Berényi, K. Vad, G. A. Langer, E. Tóth-Kádár, J. Pádár,
L. Pogány, and I. Bakonyi, Electrochim. Acta, 53, 837 (2007).

2. G. L. Katona, Z Berényi, L. Péter, and K. Vad, Vacuum, 82, 270 (2007).

13. D. Gupta, A. C. Nayak, M. Sharma, R. R. Singh, S. K. Kulkarni, and R. K. Pandey, Thin Solid Films, 513, 187 (2006).

14. M. Alper, W. Schwarzacher, and S. J. Lane, J. Electrochem. Soc., 144, 2346 (1997).

15. S. K. J. Lenczowski, C. Schönenberger, M. A. M. Gijs, and W. J. M. de Jonge, $J$. Magn. Magn. Mater, 148, 455 (1995).

16. L. Péter, Á. Cziráki, L. Pogány, Z. Kupay, I. Bakonyi, M. Uhlemann, M. Herrich, B. Arnold, T. Bauer, and K. Wetzig, J. Electrochem. Soc., 148, C168 (2001).

17. M. Alper, Lect. Notes Phys., 593, 111 (2002).

18. M. Shima, L. Salamanca-Riba, R. D. McMichael, and T. P. Moffat, J. Electrochem. Soc., 149, C439 (2002).

19. Á. Cziráki, L. Péter, V. Weihnacht, J. Tóth, E. Simon, J. Pádár, L. Pogány, C. M. Schneider, T. Gemming, K. Wetzig, et al., J. Nanosci. Nanotechnol., 6, 2000 (2006).

20. I. Bakonyi, L. Péter, Z. E. Horváth, J. Pádár, L. Pogány, and G. Molnár, J. Electrochem. Soc., 155, D688 (2008).

21. L. Péter, J. Pádár, E. Tóth-Kádár, Á. Cziráki, P. Sóki, L. Pogány, and I. Bakonyi, Electrochim. Acta, 52, 3813 (2007)

22. M. Shima, L. G. Salamanca-Riba, R. D. McMichael, and T. P. Moffat, J. Electrochem. Soc., 148, C518 (2001).

23. G. Nabiyouni and W. Schwarzacher, J. Cryst. Growth, 275, e1259 (2005).

24. K. Liu, K. Nagodawithana, P. C. Searson, and C. L. Chien, Phys. Rev. B, 51, 7381 (1995).

25. C. Schimpf, Diplomarbeit, Technische Universität Bergakademie Freiberg, Germany (2007).

26. S. Valizadeh, E. B. Svedberg, and P. Leisner, J. Appl. Electrochem., 32, 97 (2002)

27. Á. Cziráki and G. Tichy, Z. Metallkd., 96, 1024 (2005).

28. S. Z. Hua, L. Salamanca-Riba, L. H. Bennett, L. J. Schwarzendruber, R. D. McMichael, D. S. Lashmore, and M. Schlesinger, Scr. Metall. Mater, 33, 1643 (1995).

29. P. Nallet, E. Chassaing, M. G. Walls, and M. J. Hÿtch, J. Appl. Phys., 79, 6884 (1996).

30. E. Tóth-Kádár, L. Péter, T. Becsei, J. Tóth, L. Pogány, T. Tarnóczi, P. Kamasa, I. Bakonyi, G. Láng, Á. Cziráki, et al., J. Electrochem. Soc., 147, 3311 (2000).

31. Á. Cziráki, L. Péter, B. Arnold, J. Thomas, H. D. Bauer, K. Wetzig, and I. Bakonyi, Thin Solid Films, 424, 229 (2003).

32. L. Wang, P. Fricoteaux, K. Yu-Zhang, M. Troyon, P. Bonhomme, J. Douglade, and A. Metrot, Thin Solid Films, 261, 160 (1995).

33. T. A. Dang and T. A. Frisk, Surf. Coat. Technol., 106, 60 (1998).

34. J. Jorzick, J. Lösch, M. Kopnarski, and H. Oechsner, Appl. Phys. A: Mater. Sci. Process., 78, 655 (2004).

35. J. S. Sabine Becker and H.-J. Dietze, Int. J. Mass Spectrom., 197, 1 (2000).

36. I. V. Veryovkin, W. F. Calaway, C. E. Tripa, and M. Pellin, Nucl. Instrum. Methods Phys. Res. B, 261, 508 (2007).

37. Y. Chen and W. Huang, Meas. Sci. Technol., 15, 2005 (2004).

38. W. Schwarzacher, J. Phys.: Condens. Matter, 16, R859 (2004).

39. For the details of the program, see http://gwyddion.net, last accessed Sep 2008.

40. D. Gupta, A. C. Nayak, D. Kaushik, and R. K. Pandey, J. Phys. Chem Solids, 66 861 (2005).

41. M. C. Lafouresse, P. J. Heard, and W. Schwarzacher, Phys. Rev. Lett., 98, 236101 (2007).

42. N. M. Hasan, J. J. Mallet, S. G. dos Santos Filho, A. A. Pasa, and W. Schwarzacher, Phys. Rev. B, 67, 081401(R) (2003).

43. R. C. Da Silva and W. Schwarzacher, J. Electrochem. Soc., 154, D88 (2007).

44. C. Bonhôte and D. Landolt, Electrochim. Acta, 42, 2407 (1997).

45. Á. Cziráki, I. Gerőcs, B. Fogarassy, B. Arnold, M. Reibold, K. Wetzig, E. TóthKádár, and I. Bakonyi, Z. Metallkd., 88, 781 (1997).

46. Á. Cziráki, J.-G. Zheng, A. Michel, Z. Czigány, G. Nabiyouni, W. Schwarzacher, E. Tóth-Kádár, and I. Bakonyi, Z. Metallkd., 90, 278 (1999).

47. S. Kainuma, S. Ishikura, K. Hisatake, T. Watanabe, and N. Fukumuro, J. Magn Soc. Jpn., 22, 224 (1998).

48. V. Holý, U. Pietsch, and T. Baumach, High-Resolution X-Ray Scattering from Thin Films and Multilayers, p. 204, Springer-Verlag, Berlin (1999). 